Understanding the importance of the temperature dependence of viscosity on the crystallization dynamics in the $\mathrm{Ge}_{2} \mathrm{Sb}_{2} \mathrm{Te}_{5}$ phase-change material

A. Aladool, M. M. Aziz, and C. D. Wright

Citation: Journal of Applied Physics 121, 224504 (2017); doi: 10.1063/1.4985282

View online: http://dx.doi.org/10.1063/1.4985282

View Table of Contents: http://aip.scitation.org/toc/jap/121/22

Published by the American Institute of Physics

\section{AP| Journal of A1Pplied Physics}

Save your money for your research.

It's now FREE to publish with us no page, color or publication charges apply.
Publish your research in the

Joumal of Applied Physics

to claim your place in applied

physics history. 


\title{
Understanding the importance of the temperature dependence of viscosity on the crystallization dynamics in the $\mathrm{Ge}_{2} \mathrm{Sb}_{2} \mathrm{Te}_{5}$ phase-change material
}

\author{
A. Aladool, M. M. Aziz, and C. D. Wright \\ College of Engineering, Mathematics and Physical Sciences, University of Exeter, Exeter EX4 4QF, \\ United Kingdom
}

(Received 30 January 2017; accepted 29 May 2017; published online 14 June 2017)

\begin{abstract}
The crystallization dynamics in the phase-change material $\mathrm{Ge}_{2} \mathrm{Sb}_{2} \mathrm{Te}_{5}$ is modelled using the more detailed Master equation method over a wide range of heating rates commensurate with published ultrafast calorimetry experiments. Through the attachment and detachment of monomers, the Master rate equation naturally traces nucleation and growth of crystallites with temperature history to calculate the transient distribution of cluster sizes in the material. Both the attachment and detachment rates in this theory are strong functions of viscosity, and thus, the value of viscosity and its dependence on temperature significantly affect the crystallization process. In this paper, we use the physically realistic Mauro-Yue-Ellison-Gupta-Allan viscosity model in the Master equation approach to study the role of the viscosity model parameters on the crystallization dynamics in $\mathrm{Ge}_{2} \mathrm{Sb}_{2} \mathrm{Te}_{5}$ under ramped annealing conditions with heating rates up to $4 \times 10^{4} \mathrm{~K} / \mathrm{s}$. Furthermore, due to the relatively low computational cost of the Master equation method compared to atomistic level computations, an iterative numerical approach was developed to fit theoretical Kissinger plots simulated with the Master equation system to experimental Kissinger plots from ultrafast calorimetry measurements at increasing heating rates. This provided a more rigorous method (incorporating both nucleation and growth processes) to extract the viscosity model parameters from the analysis of experimental data. The simulations and analysis revealed the strong coupling between the glass transition temperature and fragility index in the viscosity and crystallization models and highlighted the role of the dependence of the glass transition temperature on the heating rate for the accurate estimation of the fragility index of phase-change materials from the analysis of experimental measurements. Published by AIP Publishing.
\end{abstract}

[http://dx.doi.org/10.1063/1.4985282]

\section{INTRODUCTION}

Modelling the crystallization dynamics of phase-change materials under fast annealing conditions is of importance to understand the requirements for achieving high data rates during the write and erase processes in optical and electronic memories. ${ }^{1}$ While quenching from the melt and re-amporphization is a relatively fast process, crystallization from the amorphous phase remains the time limiting process in phase-change based memories. ${ }^{2}$ The kinetic and thermodynamic parameters of the material, in addition to the viscosity dependence on temperature, directly affect the speed of crystallization of the phasechange material. Therefore, understanding the role of these parameters on the crystallization process through modelling and simulation is crucial for the development of modern, highspeed phase-change memory devices and technologies.

The crystallization process has been modelled using different approaches including the analytical and well-known model of Johnson-Mehl-Avrami-Kolmogorov (JMAK). ${ }^{3,4}$ This model considers nucleation being random and uniform at constant (isothermal) temperature. However, these assumptions may not be applicable since crystallization in phase-change materials due to nucleation is not random nor uniform, ${ }^{5}$ and the nucleation rate cannot be considered time independent for the entire crystallization process with temperature history. Nucleation and growth are separately considered in the classical nucleation and growth model. ${ }^{6}$ This model only deals with crystal clusters exceeding the critical size $^{7}$ and therefore ignores transient subcritical cluster formation responsible for modelling the incubation time. Another approach for studying the crystallization process in phase-change materials at the fundamental level is ab-initio atomic scale modelling which depends on the density functional theory (for example, Ref. 8). Although this approach can provide deep insights into the crystallization behaviour in phase-change materials, these simulations are too computationally expensive for the analysis of experimental data or device level modelling.

One of the approaches for bridging the gap between the large-scale, simplified analytical models (such as the JMAK description) and the first-principle and numerically intensive atomistic modelling is the robust and physically realistic Master rate equation method considered in this work. Nucleation and growth in this model are described by the attachment and detachment of monomers, ${ }^{5}$ yielding transient cluster size distributions in the subcritical and supercritical regimes under isothermal and non-isothermal annealing conditions. ${ }^{9,11}$ This method has been successfully used to model crystallization in phase-change materials ${ }^{10-12}$ and simulation of differential calorimetry for glasses ${ }^{9}$ and offer relatively faster computation times compared to atomistic simulations for practical cluster sizes. The rates of attachment and detachment in the Master equation model, which control the 
speed of crystallization, are functions of the temperature dependence of the viscosity, and hence, the simulations are sensitively characterized by the viscosity model.

A number of models have been developed to describe the viscosities of glasses in a broad range of chemical compositions and temperatures. ${ }^{13}$ The Arrhenius viscosity mode ${ }^{14}$ is commonly used in investigations of crystallization dynamics in $\mathrm{Ge}_{2} \mathrm{Sb}_{2} \mathrm{Te}_{5}$ (GST). ${ }^{11,15}$ However, extracted viscosities for GST from ultrafast differential scanning calorimetry (DSC) measurements and growth rate measurements showed deviation from the Arrhenius temperature dependence, particularly demonstrating a fragile behaviour in the supercooled region. ${ }^{16,17}$ Other non-Arrhenius models for viscosity in phase-change materials have been adopted including the Cohen and Grest viscosity model and the threeparameter models. The Cohen and Grest viscosity model has four adjustable parameters to fit measured viscosity data. ${ }^{18}$ This model was used in Ref. 16 to extract the dependence of viscosity and crystal growth rates on temperature from ultrafast DSC measurements for GST. The fitting constants in this viscosity model are not related to physical parameters of viscosity (such as the fragility and glass transition temperature). It was also noted that the Cohen and Grest viscosity model over-estimated crystal growth rates in $\mathrm{Ge}-\mathrm{Sb}$ alloys by $2-3$ orders of magnitude compared to the three-parameter models $^{19}$ and was not able to provide accurate fitting to Kissinger plots at high heating rates for the AgInSbTe phasechange material. ${ }^{20}$

The three-parameter viscosity models include the wellknown Vogel-Fulcher-Tammann (VFT) model, ${ }^{21}$ the Avramov-Milchev (AM) model, ${ }^{22}$ and the widely accepted Mauro-Yue-Ellison-Gupta-Allan (MYEGA) model. ${ }^{23}$ The VFT model has three fitting parameters and was used to describe the temperature dependence of viscosity in GST (for example, Refs. 17 and 24). The more robust MYEGA viscosity model has physical foundation with three physical parameters including the glass transition temperature $T_{g}$, fragility $m$, and extrapolated infinite temperature viscosity $\eta_{\infty}{ }^{23}$ This model was successfully used to describe the temperature dependence of viscosity and crystal growth rates for AgInSbTe (AIST), ${ }^{25,26} \mathrm{GeSb},{ }^{19}$ and GST. ${ }^{27}$ The extended, five parameter generalised MYEGA model ${ }^{28}$ was also used to describe the fragile-to-strong cross-over of viscosity in the AgInSbTe phase-change material (which is not apparent in GST) ${ }^{20}$ This paper is therefore concerned with implementing the MYEGA model for the viscosity dependence on temperature in the Master equation system to simulate crystallization in GST at high heating rates.

The fragility index $m$ in the MYEGA viscosity model represents the slope of the viscosity in the Angell plot at the glass transition temperature and indicates the degree of deviation from the Arrhenius temperature dependence of viscosity. For the GST phase-change material, the fragility index has been extracted from fitting simplified (growth dominated) JMAK formulation with the VFT viscosity model to Kissinger plots measured using ultrafast differential scanning calorimetry (DSC), with a reported value of $\sim 90 .{ }^{16}$ Mechanical stress measurements on GST films at relatively low heating rates (assuming the Arrhenius temperature dependence of viscosity and a heating rate dependent $T_{g}$ ) indicated fragilities of 47 and 20 for pure and doped GST, respectively. ${ }^{17}$ Higher values of fragility of 140 were also estimated from fitting to device level measurements of crystal growth rates as a function of temperature. ${ }^{27}$ There is thus dispersion and uncertainty in the extracted values of fragility, but they all indicate the fragile nature of GST, which contributes to the high atomic mobility and fast crystallization of this phase-change material when heated at the relevant temperatures.

Published values of the glass transition temperature $T_{g}$ for GST also vary, with $373 \mathrm{~K}$ reported for thin (as-deposited) amorphous films using impedance, transmission, and heat capacity measurements. ${ }^{29}$ This value is in agreement with a glass transition temperature of $384 \mathrm{~K}$ determined from the theory based on the enthalpy of atomization for GST ${ }^{30}$ and was therefore adopted in the theoretical analysis and fitting to measured Kissinger plots in ultrafast DSC simulations in Ref. 16. However, DSC measurements of pre-annealed thin amorphous films of the phase-change material at relatively low heating rates $(40 \mathrm{~K} / \mathrm{min})$ revealed higher glass transition temperatures within $10 \mathrm{~K}$ of the peak crystallization temperature (456 K for GST) and hence difficult to resolve from the main crystallization peak in the measurement. ${ }^{31}$ The highest reported value of $T_{g}$ for GST was estimated from fitting the MYEGA model for the viscosity to crystal growth velocity measurements at the device level and was $472 \mathrm{~K} .{ }^{27}$ The infinite temperature viscosity parameter, $\eta_{\infty}$, can be extracted from Angell plots extrapolated to high temperatures and is typically in the range of $10^{-5}-10^{-3}$ (Pa s). ${ }^{16}$

It is clear from the brief overview of reported viscosity parameters above that there are uncertainties in the values of fragility and glass transition temperature for GST in the MYEGA model. This disparity in the estimated viscosity parameters may arise due to different types of samples (powder, flakes, and thin-films), preparation conditions, preannealing, heating rates, and more fundamentally the nature of the crystallization and viscosity models imposed for fitting to experimental data. An example is the large difference in the value of fragility $m>100$ derived for AIST from fitting the MYEGA model to growth velocity measurements, ${ }^{25}$ compared to the value of $m \sim 37$ derived for the same material using the generalised MYEGA model when accounting for the fragile-to-strong transition behaviour of the viscosity. ${ }^{20}$ Furthermore, current methods of extraction of the viscosity parameters often rely on simple models of crystallization, such as the JMAK model with the constant nucleation rate and growth dominated crystallization which may not be appropriate for nucleation dominated materials such as GST. In this paper, we use the more detailed Master equation approach which includes both transient nucleation and growth processes with viscosity described by the MYEGA model to study the crystallization dynamics of GST over a wide range of heating rates. In particular, we investigate the role of the viscosity model and its parameters on the crystallization dynamics using Master equation simulations and use this model to simulate DSC measurements and full Kissinger plots. Due to its practical computational times, we also developed an iterative numerical algorithm 
based on the physically realistic Master rate equation to estimate the values of the viscosity parameters for GST in comparison to published Kissinger plots from ultrafast DSC measurements. In this analysis, we particularly highlight and explore the effects of the dependence of glass transition temperature on the heating rate on the extracted fragility values and the strong correlation between the glass transition temperature and fragility index.

Section II of this paper will describe the theory of cluster formation thermodynamics in phase-change materials, leading to the mathematical formulation of the Master equation approach and the viscosity model used in the simulations. This is followed by the results and simulations section, illustrating the effects of the parameters of the MYEGA viscosity model on the crystallization dynamics in GST. The development of the iterative Master equation algorithm and comparison with published DSC measurements for GST at ultrafast heating rates is detailed in Section III, followed by discussion of the outcomes of this article and main conclusions.

\section{THEORY}

\section{A. Cluster formation thermodynamics}

The mechanism of attachment and detachment of monomers in the nucleation theory depends on the required work for the heterogeneous formation of clusters through attachment of monomers up to the theoretical cluster size, and then, they can grow rather than dissociate. The work for cluster formation, denoted by $W(n, T)$, is the sum of a surface free energy and a bulk free energy at certain temperature $T$ and is written as

$$
W(n, T)=-K_{1}(T) n+K_{2} n^{2 / 3},
$$

where $n$ is the number of monomers and $K_{1}$ is the difference in the bulk Gibbs free energy between the old and new phases, which may be calculated from ${ }^{32}$

$$
K_{1}=\sigma_{0} \Delta H_{f}\left(\frac{T_{m}-T}{T_{m}}\right)\left[\frac{7 T}{T_{m}+6 T}\right],
$$

where $\sigma_{0}$ is the volume of a monomer (bonding molecule), $T_{m}$ is the melting temperature of the phase-change material, and $\Delta H_{f}$ is the enthalpy of fusion at the melting point. The difference in the surface energy between the two phases can be described by the interfacial energy coefficient $K_{2}$, which may be written as ${ }^{5}$

$$
K_{2}=a \sigma\left[\varphi\left(\theta_{w}\right)\right]^{\frac{2}{3}},
$$

where $a=\left(36 \pi \sigma_{0}^{2}\right)^{\frac{1}{3}}$ is the surface area for spheres, $\sigma$ is the interfacial energy, and $\varphi\left(\theta_{w}\right)$ is the spherical cap geometrical factor to model the effects of heterogeneous nucleation with wetting angle $\theta_{w}$ (from the substrate surface) ${ }^{5}$

$$
\varphi\left(\theta_{w}\right)=\frac{1}{4}\left(2+\cos \theta_{w}\right)\left(1-\cos \theta_{w}\right)^{2},
$$

where $0<\theta_{w} \leq \pi$ to model homogeneous $\left(\theta_{w} \rightarrow \pi\right)$ and heterogeneous $\left(\theta_{w} \rightarrow 0\right)$ nucleation.

It can be seen from (2) that the sign of $K_{1}$ changes at $T=T_{m}$ and below the melting temperature $K_{1}>0$, indicating a maximum whose position is determined from

$$
\frac{\partial W(n, T)}{\partial n}=0
$$

yielding

$$
n^{*}=\frac{8}{27}\left(\frac{K_{1}}{K_{2}}\right)^{3} .
$$

This is the critical size when the crystal cluster is likely to continue to grow rather than dissociate. The cluster formation thermodynamics described in this section provide the driving force for crystallization in the Master rate equation formulation described next.

\section{B. The Master rate equation}

The Master rate equation system is described here for completeness following the development by Kashchiev ${ }^{5}$ consistently, which uses different attachment and detachment rates compared to previous work in Refs. 9 and 11. The dynamics of crystallization in phase-change materials is modelled by describing the transient evolution of the density of clusters of size $n$ monomers, $Z$, per unit volume with temperature $T$ in the Master equation

$$
\begin{aligned}
\frac{d}{d t} Z(n, t)= & f(n-1, t, T) Z(n-1, t) \\
& +g(n+1, t, T) Z(n+1, t)-f(n, t, T) Z(n, t) \\
& -g(n, t, T) Z(n, t), \quad n \geq 2,
\end{aligned}
$$

where $f(n, t, T)$ and $g(n, t, T)$ are the attachment and detachment rates respectively. ${ }^{5}$ The detachment rate may be expressed in terms of the attachment rate following Zeldovich for $(n=1,2, \ldots):^{33,34}$

$$
f(n, t, T) C(n, T)=g(n+1, t, T) C(n+1, T),
$$

where $C(n, T)$ is the quasi-equilibrium cluster size distribution which can be expressed thermodynamically as ${ }^{5}$

$$
C(n, T)=\exp \left(-W(n, T) / k_{B} T\right)
$$

The attachment and detachment rates are given respectively by $^{5}$

$$
\begin{gathered}
f(n, t, T)=Z_{m} \hat{f} n^{2 / 3} \exp \left(\frac{(W(n, T)-W(n+1, T))}{k_{B} T}\right), \\
g(n, t, T)=f(n-1, t, T) \frac{C(n-1, T)}{C(n, T)}
\end{gathered}
$$

where $k_{B}$ is the Boltzmann constant and $Z_{m}$ is the density of the remaining amorphous monomers, which is computed from 


$$
Z_{m}=Z_{0}-\sum_{n=2}^{n_{\max }} n Z(n, t)
$$

where $Z_{0}$ is the total monomer density of the starting (amorphous) phase. Thus, the volume fraction of the crystallised material can be computed from (12) as

$$
\chi=\sum_{n=2}^{n_{\max }} n Z(n, t) / Z_{0} .
$$

It is noted that the detachment rate in (11) is independent of the amount of free monomers $Z_{m}$. In (10) and (11), $\hat{f}$ is given by

$$
\hat{f}=\frac{c \sigma_{0}^{2 / 3}\left(1-\cos \theta_{w}\right)}{2 d_{0} \varphi\left(\theta_{w}\right)^{2 / 3}} D,
$$

where $d_{0}$ is the diameter of a monomer, $c=(36 \pi)^{1 / 3}$ is the shape factor for heterogeneous nucleation, and $D$ is the selfdiffusion coefficient which may be described by the StokesEinstein equation

$$
D=\frac{k_{B} T}{3 \pi \lambda \eta(T)} .
$$

In (15), $\lambda$ is the jump distance which is taken to be equal to the interatomic distance in GST $(2.99 \AA),{ }^{35}$ and $\eta(T)$ is the temperature dependant viscosity. Thus, as indicated in (10) and (11), both the attachment and detachment rates, respectively, and therefore crystallization dynamics, are strongly influenced by the functional dependence of viscosity on temperature.

\section{Viscosity model}

In this section, both the Arrhenius and non-Arrhenius (MYEGA) models of the viscosity dependence on temperature are presented and used to derive the complete viscosity model from room temperature up to the melting point used in the subsequent Master equation simulations. In the Arrhenius model, the viscosity is described by

$$
\eta(T)=K_{\eta} \exp \left[\frac{E_{a}}{k_{B} T}\right],
$$

where $K_{\eta}$ is a prefactor and $E_{a}$ is the activation energy for viscous flow. This model has been previously employed in reaction rate modelling of crystallization in phase-change materials and yielded good agreement between simulations and measurements for isothermal annealing and at low heating rates. ${ }^{9,11}$ Recent ultra-high heating rate DSC measurements, however, indicated the deviation of the viscosity from the Arrhenius behaviour for GST ${ }^{16}$ and other phase-change materials including AIST $^{25,26}$ and GeSb. ${ }^{19}$

Therefore, in this work, we focus on the more robust and widely accepted viscosity model of Mauro-Yue-EllisonGupta-Allan (MYEGA). ${ }^{23}$ This model has physical grounding and is able to describe different viscosity behaviours over a wide temperature range from $T_{g}$ (glass transition temperature) to $T_{m}$ (melting temperature) and is given by

$$
\begin{aligned}
\log _{10} \eta(T)= & \log _{10} \eta_{\infty}+\left(12-\log _{10} \eta_{\infty}\right) \frac{T_{g}}{T} \exp \\
& \times\left[\left(\frac{m}{\left(12-\log _{10} \eta_{\infty}\right)}-1\right)\left(\frac{T_{g}}{T}-1\right)\right],
\end{aligned}
$$

for $T \geq T_{g}$. The three parameters in the MYEGA model are as follows: $\eta_{\infty}$ is the extrapolated infinite temperature viscosity, $T_{g}$ is the glass transition temperature at which the shear viscosity is equal to $10^{12}(\mathrm{~Pa} \mathrm{~s}),{ }^{36}$ and $m$ is the fragility index of the material defined as

$$
m={\frac{\partial \log _{10}(\eta(T))}{\partial\left(T_{g} / T\right)}}_{T=T_{g}} .
$$

The fragility index is the slope of the viscosity curve at the glass transition temperature in the Angell plot and indicates the degree of deviation from the Arrhenius behaviour in the supercooled region.

The MYEGA model is also able to describe the viscosity of a wide class of phase-change materials and behaviours including the Arrhenius behaviour. This can be illustrated by taking the logarithm on both sides of (16) to yield

$$
\log _{10}(\eta(T))=\log _{10} K_{\eta}+\left[\frac{E_{a} \log _{10}(e)}{k_{B} T_{g}}\right] \times \frac{T_{g}}{T} .
$$

By comparing (17) to (19), it can be readily shown that the MYEGA model can describe the Arrhenius temperature dependence provided that

$$
\log _{10} K_{\eta}=\log _{10} \eta_{\infty}
$$

and

$$
12-\log _{10} \eta_{\infty}=\left[\frac{E_{a} \log _{10}(e)}{k_{B} T_{g}}\right],
$$

while the exponential term in (17) equates to

$$
\exp \left[\left(\frac{m}{\left(12-\log _{10} \eta_{\infty}\right)}-1\right)\left(\frac{T_{g}}{T}-1\right)\right]=1 .
$$

The above equalities show that through appropriate choice of the three parameters, the MYEGA model can describe the strong, Arrhenius temperature dependence of viscosity near the glass transition temperature for small fragilities where $m \rightarrow\left(12-\log _{10} \eta_{\infty}\right)$.

For temperatures $T<T_{g}$, we have described the viscosity dependence on temperature using the Arrhenius model defined in (16), with the activation energy $E_{a}=1.76$ $\pm 0.05 \mathrm{eV}$ measured by Kalb et al. for GST in Ref. 37 . The prefactor $K_{\eta}$ in (16) in this case was derived here from the requirement that $\log _{10} \eta(T)=12$ at $T=T_{g}$ to match the viscosity of the MYEGA model. Hence, the complete viscosity model derived in this work is given by

$$
\log _{10} \eta(T)= \begin{cases}12+\log _{10}\left(\exp \left[\frac{E_{a}}{k_{B}}\left(\frac{1}{T}-\frac{1}{T_{g}}\right)\right]\right) & T<T_{g} \\ \text { MYEGA } & T \geq T_{g} .\end{cases}
$$


Figure 1 illustrates the temperature dependence of viscosity calculated using the MYEGA model for different values of fragility. The viscosity model in (21) is used in the subsequent simulations in this paper.

\section{SIMULATION RESULTS}

In this section, transient simulations of crystallization over a wide range of heating rates are carried out by solving the Master rate equation to determine the key parameters within the MYEGA viscosity model that affect the crystallization dynamics. Furthermore, an iterative numerical algorithm based on the Master equation method is developed to compare simulated and measured Kissinger plots from experimental calorimetry studies published in the literature so as to extract the viscosity model parameters and examine the role of the viscosity and crystallization models on the extracted values.

The system of coupled equations in (7) was solved numerically for the cluster size distribution $Z(n, t)$ using the ode15s solver in Matlab, with absolute and relative tolerances of $10^{-10}$ (to provide convergence to a stable and accurate solution with practical computation time). The solution tracks the formation and destruction of clusters of size $n \geq 2$ ( $n=1$ representing the amorphous phase) with temperature history. The initial starting phase in all simulations was the amorphous phase with initial cluster size distribution $Z(n, 0)$ $\rightarrow 0$ ( $10^{-11}$ clusters $/ \mathrm{m}^{3}$ used here). Starting from room temperature $T_{0}=300 \mathrm{~K}$, the numerical simulations were performed over increasing heating rates $\phi$ from $50 \mathrm{~K} / \mathrm{s}$ up to $40000 \mathrm{~K} / \mathrm{s}$ according to $T=T_{0}+\varphi t$ where $t$ is the time, to allow comparison with previously published ultrafast DSC measurements for GST. ${ }^{16,19}$ The maximum number of equations solved in (7) (upper limit of cluster size) was 40, which is greater than the critical size of clusters over the temperature range $(300 \mathrm{~K}$ to $635 \mathrm{~K})$ used in this work, to allow accurate simulations of crystallization. ${ }^{9}$ This was confirmed by carrying out simulations with increasing cluster sizes up to 1000 monomers at heating rates up to the $40000 \mathrm{~K} / \mathrm{s}$, showing particularly negligible changes in the transient response

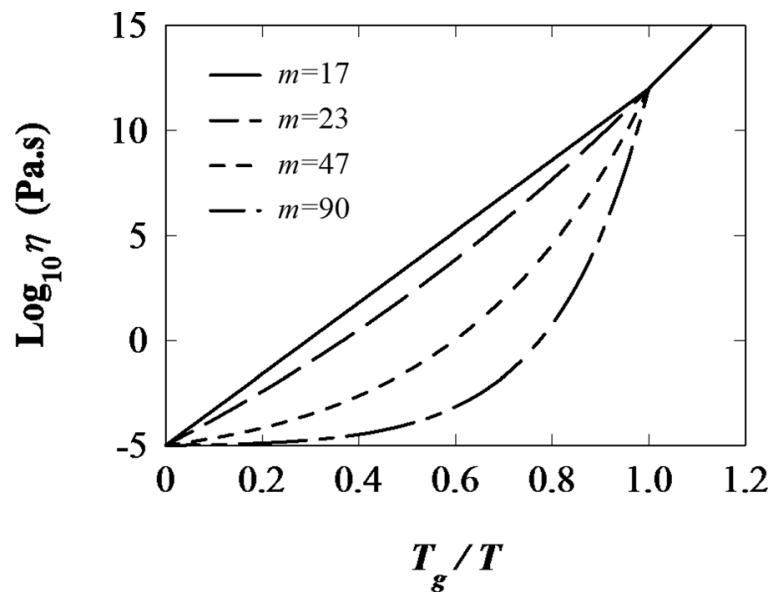

FIG. 1. Angell plot for the temperature dependence of calculated viscosity using the complete viscosity model described in (21) with parameter values: $\eta_{\infty}=10^{-5}$ (Pa s), $T_{g}=383 \mathrm{~K}$, and $E_{a}=1.76 \mathrm{eV}$ in the Arrhenius model for $T<T_{g}$. and in peak crystallization temperatures with increasing cluster size (important in this work for accurate fitting to experimental Kissinger plots). The only noticeable difference was a $16 \%$ maximum increase in the steady-state crystalline volume fraction happening only at the highest heating rate as the maximum cluster size was increased from 40 to 1000 monomers. These simulations confirmed the validity of using a maximum cluster size of 40 , which is larger than the maximum critical cluster size for the temperature range used in the simulations, enabling quick computational times and fitting to experimental measurements.

The thermodynamic and material parameters used in the simulations are listed in Table I. The surface energy and glass transition temperatures were varied in the simulations within the range of reported values in the literature to investigate their effect (along with the fragility index) on the transient crystallization behaviour.

Figure 2(a) shows an example of the cluster size distribution from annealing at a heating rate of $50 \mathrm{~K} / \mathrm{s}$ calculated from the solution of the Master equation using the parameters listed in Table I at different instances of time. This figure shows the formation and evolution of larger clusters during nucleation and growth following the initial amorphous phase. Figure 2(b) shows the distribution of cluster sizes at the final iteration in which the volume is fully crystallized.

\section{A. The effects of viscosity parameters on crystallization dynamics}

The fragility index, the glass transition temperature, and the infinite temperature viscosity are the main parameters which describe the temperature dependence of viscosity in the MYEGA model and influence the rates of attachment and detachment of monomers (and therefore crystal nucleation and growth rates) in the Master rate equation. We will next study the effects of these parameters on the crystallization dynamics using the Master equation simulations, in particular, on the transient rate of crystallization and on the peak crystallization temperature $T_{p}$ - defined here as

TABLE I. Thermodynamic and material parameters used for the numerical simulations.

\begin{tabular}{lcc}
\hline \hline Parameter & Value & Unit \\
\hline Volume of a monomer $\sigma_{0}$ & $2.9 \times 10^{-28 \mathrm{a}}$ & $\mathrm{m}^{-3}$ \\
Enthalpy of fusion $\Delta H_{\mathrm{f}}$ & $6.18 \times 10^{8 \mathrm{a}}$ & $\mathrm{J} / \mathrm{m}^{3}$ \\
Melting temperature $T_{\mathrm{m}}$ & $889^{\mathrm{b}}$ & $\mathrm{K}$ \\
Specific surface energy $\sigma$ & $0.033-0.066^{\mathrm{c}}$ & $\mathrm{J} / \mathrm{m}^{2}$ \\
Wetting angle $\theta_{\mathrm{w}}$ & $100^{\mathrm{d}}$ & $\mathrm{Deg}$ \\
Glass transition temperature $T_{\mathrm{g}}$ & $383-472^{\mathrm{e}}$ & $\mathrm{K}$ \\
Infinite temperature viscosity $\eta_{\infty}$ & $1 \times 10^{-5 \mathrm{f}}$ & $(\mathrm{Pa} \mathrm{s})$ \\
\hline \hline
\end{tabular}

${ }^{\mathrm{a}}$ References 10 and 11.

${ }^{\mathrm{b}}$ Reference 38 .

${ }^{\mathrm{c}}$ The surface energy for GST is within published values in Ref. 39.

${ }^{\mathrm{d}}$ Reference 11; typical value for mostly homogeneous nucleation in powder samples $^{16}$ and includes a contribution of heterogeneous nucleation to account for potential surface crystallization in the experimental DSC samples that we are comparing with in this work.

${ }^{\mathrm{e}}$ References 16, 27, 29, and 31.

${ }^{\mathrm{f}}$ Extrapolated from Ref. 16 for temperatures $T \geq T_{m}$. 


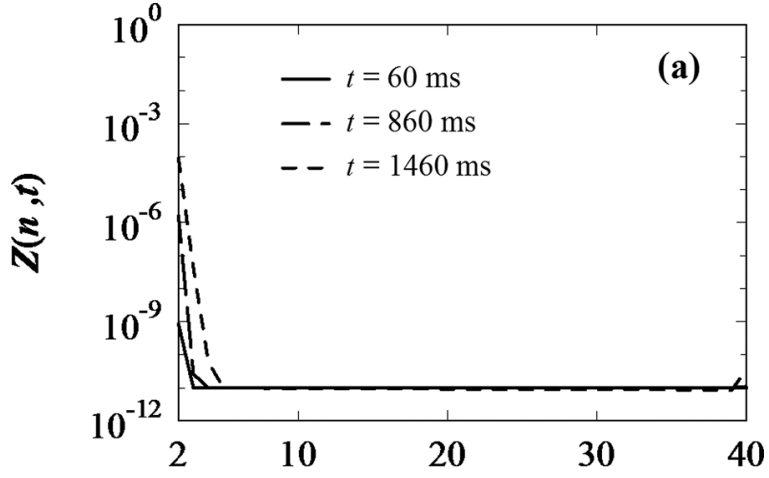

Cluster size, $n$

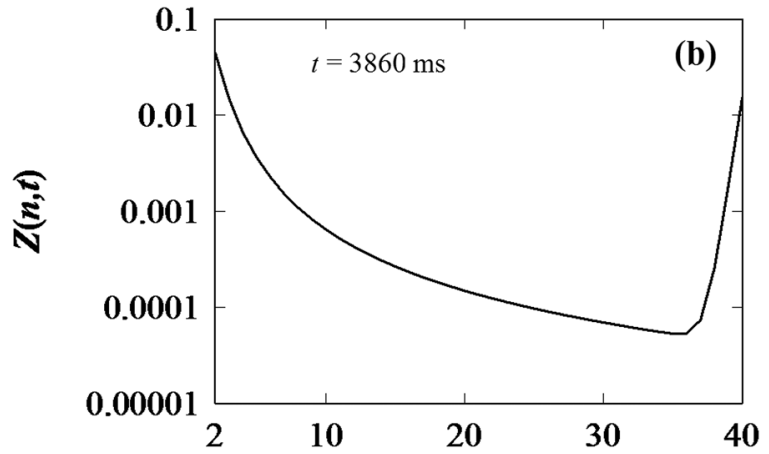

Cluster size, $\boldsymbol{n}$

FIG. 2. Cluster size evolution with time calculated from the numerical solution of the Master equation for ramped annealing at a constant heating rate of $50 \mathrm{~K} / \mathrm{s}$. (a) Initial iterations. (b) Cluster size distribution at the final iteration. Viscosity model parameters used in the simulations include $m=23$ and $T_{g}=383 \mathrm{~K}$.

temperature at which the crystallization rate is maximumat different heating rates.

Figure 3(a) shows the illustration of the effects of the fragility index on the crystalline volume fraction transformed, where low fragility values $(m=23)$ decrease the rate of crystallization and increase the peak crystallization temperature, leading to incomplete crystallization at high heating rates. This is attributed to the reduction in the diffusion coefficient (atomic mobility) in the rate equation with increasing viscosity, which increases the transient nucleation time for the clusters (time for the onset of steady-state nucleation in $Z$ ) as shown in Fig. 3(b) for relatively low heating rates $(50 \mathrm{~K} / \mathrm{s})$ and in Fig. 3(c) for the highest heating rate $(40$ $000 \mathrm{~K} / \mathrm{s})$. On the other hand, higher fragility values $(m=90)$ increase the atomic mobility in the supercooled region, therefore reducing the transient nucleation time and increasing the crystallization rates. This is illustrated by the sharp increase in the cluster size distributions for $n=3$ in Figs. 3 (b) and 3(c) around $1.7 \mathrm{~s}$ and $2 \mathrm{~ms}$, respectively, marking the sharp drop in viscosity as the temperature reaches the glass transition temperature in the simulations. In this case (higher $m$ ), full crystallization is achieved even at relatively high heating rates with the modest increase in $T_{p}$ as indicated in Fig. 3(a). It can also be observed from Figs. 3(b) and 3(c) that the transient nucleation rate of clusters of different sizes is not uniform and changes with the heating rate. In general,
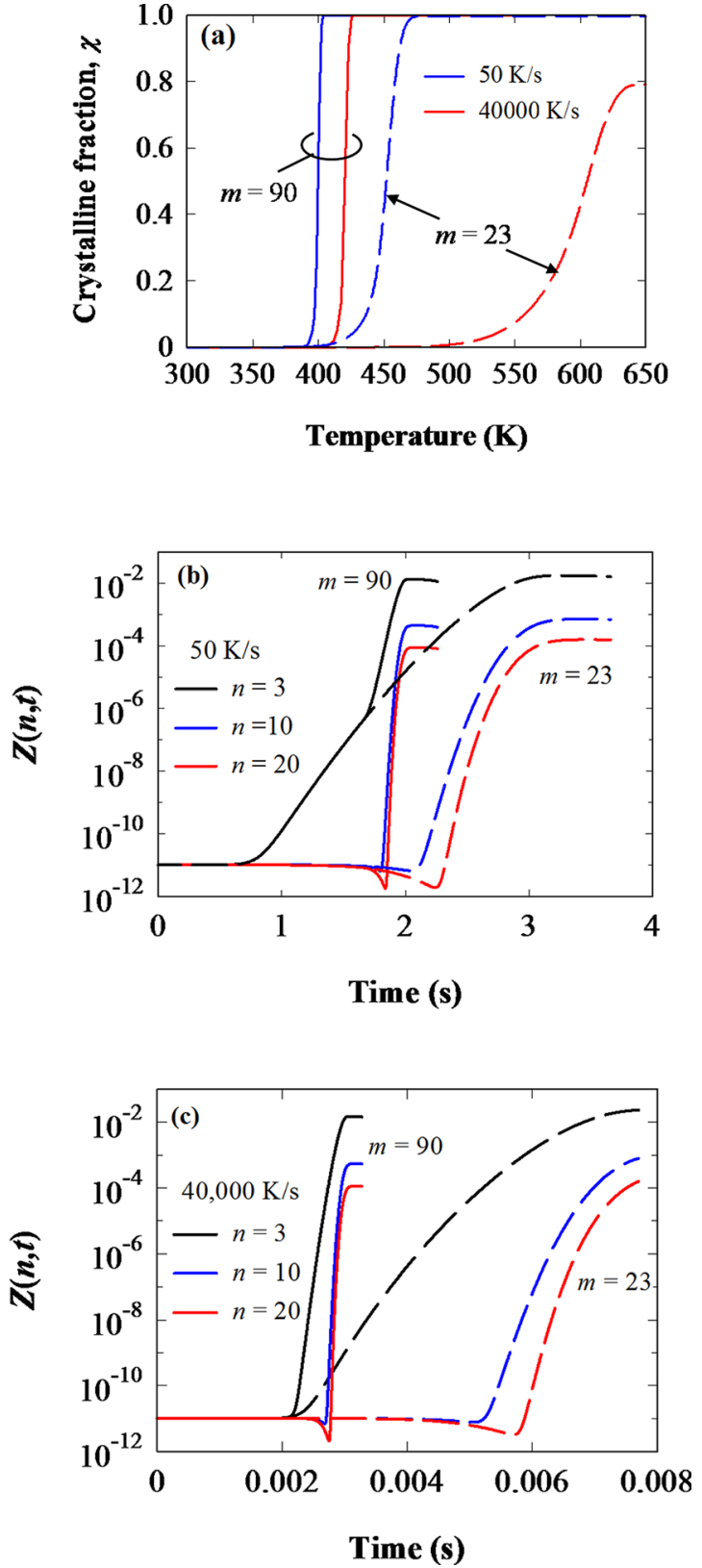

FIG. 3. (a) Calculated crystalline volume as a function of temperature during ramped anneals at different heating rates for two different fragility values: $m=90$ (solid lines) and $m=23$ (dashed lines). The calculated transient cluster densities for different cluster sizes at heating rates of (b) $50 \mathrm{~K} / \mathrm{s}$ and (c) $40000 \mathrm{~K} / \mathrm{s}$, showing non-uniform transient nucleation and the influence of the increasing fragility parameter on reducing the cluster nucleation time and increasing the crystallization speed. In these plots, the fragility values used in the calculations are $m=90$ (solid line) and $m=23$ (dashed line). Parameters used in the simulations include $T_{g}=383 \mathrm{~K}$.

Fig. 3 illustrates the influence of the increasing fragility index on reducing the cluster nucleation time and increasing the crystallization speeds.

The glass transition temperature $T_{g}$ was next varied within the range of published values shown in Table I in the Master equation simulations. The calculated crystalline volume fractions are shown in Fig. 4(a) for a strong glass with $m=23$ in the MYEGA model, illustrating an increase in $T_{p}$ followed by an increase in $T_{p}$ following the increase in $T_{g}$ (compare dashed lines versus solid lines), leading to incomplete crystallization and increased temperature difference 


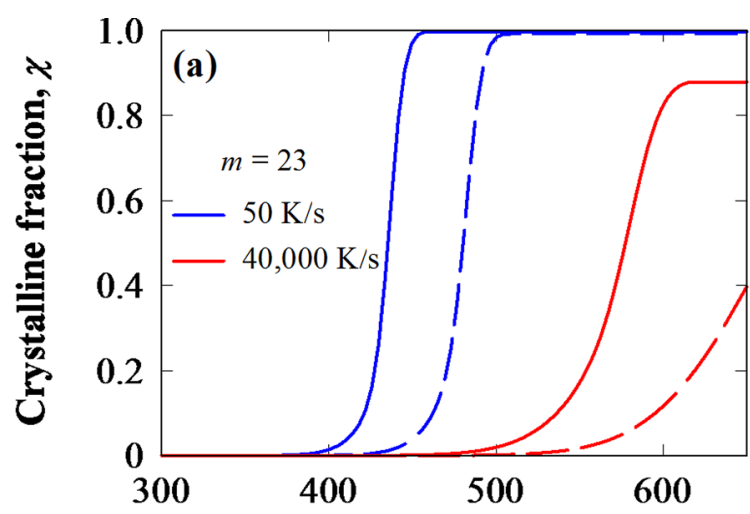

Temperature (K)

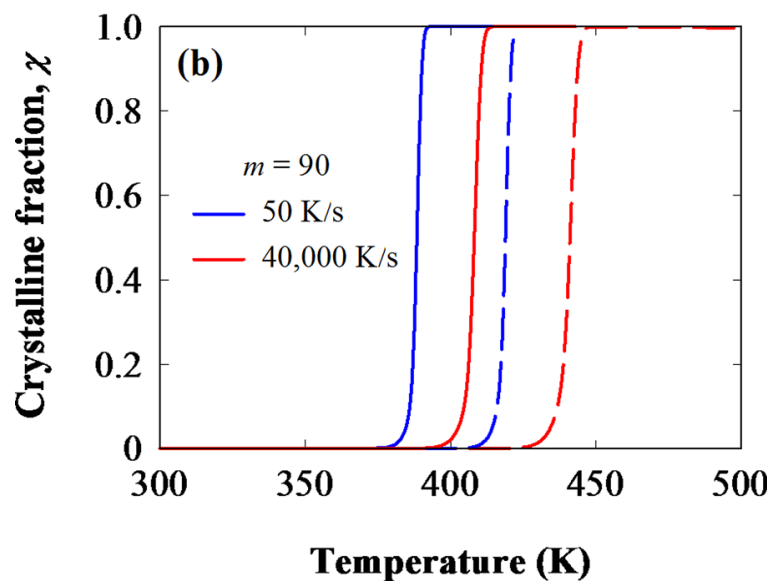

FIG. 4. Calculated crystalline volume fraction as a function of temperature during ramped anneals at different heating rates. Two different values of $T_{g}$ are used in the calculations: $T_{g}=373 \mathrm{~K}$ (solid line) and $T_{g}=400 \mathrm{~K}$ (dashed line) at different heating rates for (a) $m=23$ and (b) $m=90$.

$T_{p}-T_{g}$ at high heating rates. The crystallization simulations in Fig. 4(b) also illustrate an increase in $T_{p}$ with increasing $T_{g}$ for a fragile material with $m=90$, with the modest changes in the temperature $T_{p}-T_{g}$ with the increasing heating rate due to the high atomic mobility of the glass in this case.

The infinite temperature viscosity $\eta_{\infty}$ was varied within the range $0.012-10^{-5}(\mathrm{~Pa} \mathrm{~s})^{16,27}$ in the Master equation simulations for the fragilities $m=23$ and $m=90$ and at different heating rates. It is expected that the influence of this parameter becomes important only at high temperatures near the melting point. As shown in Fig. 5(b), varying $\eta_{\infty}$ over this large range of values has a relatively small effect on the crystallization dynamics for high fragility values in the MYEGA model, where the high diffusivities increase the crystallization rate and lower $T_{p}$ well below the melting point $T_{m}$. For low fragility values, the influence of $\eta_{\infty}$ on the crystallization dynamics depends on the heating rate (which controls $T_{p}$ ). At low heating rates, $T_{p}$ is again well below $T_{m}$ and the value of $\eta_{\infty}$ has negligible effects on the crystallization dynamics as shown in Fig. 5(a) at $50 \mathrm{~K} / \mathrm{s}$. Increasing the heating rate increases $T_{p}$ to higher temperatures towards the melting point, where smaller values of $\eta_{\infty}$ increase the crystallization rate and final crystalline volume fraction as shown in Fig. 5(a). Since the
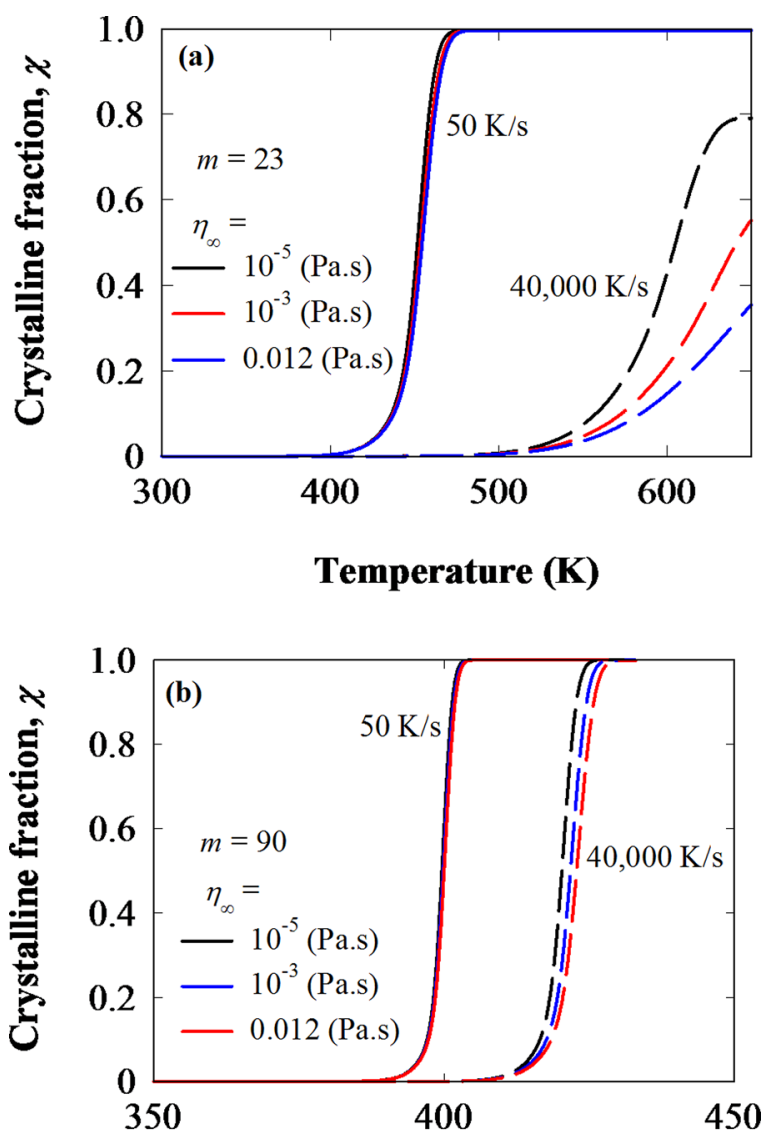

Temperature (K)

FIG. 5. Calculated crystalline volume fraction as a function of temperature during ramped anneals at different heating rates. Three different values of $\eta_{\infty}$ were used at the heating rates $50 \mathrm{~K} / \mathrm{s}$ (dashed lines) and $40000 \mathrm{~K} / \mathrm{s}$ (solid lines). $T_{g}=383 \mathrm{~K}$ was used in the simulations for the fragilities: (a) $m=23$ and (b) $m=90$.

simulations in this work occur within temperatures lower than the melting point for GST, a constant value of $\eta_{\infty}=10^{-5}(\mathrm{~Pa}$ s) was employed in this work, allowing focus on the more important fragility index and glass transition temperature as variable parameters in the DSC simulations.

\section{B. Kissinger plots and comparison to DSC measurements}

Differential scanning calorimetry (DSC) is a valuable tool to investigate crystallization dynamics and extract important kinetic and thermodynamic parameters of phase-change materials. ${ }^{40}$ The peak crystallization temperature $T_{p}$ is determined from the peaks in the measured DSC traces at different heating rates $\phi$ and used to produce Kissinger plots ${ }^{40}$ in which $\ln \left(\varphi / T_{p}^{2}\right)$ is plotted versus $1 / T_{p}$ with the plot being typically a straight line (to describe an Arrhenius behaviour), enabling the estimation of the activation energy for the reaction. ${ }^{41}$ The crystallization dynamics over a broad range of heating rates $(50 \mathrm{~K} / \mathrm{s}$ up to $40000 \mathrm{~K} / \mathrm{s}$ ) have been measured using ultrafast DSC measurements for GST (see Fig. 7) ${ }^{16,19}$ and for other phase-change materials (such as GeSb ${ }^{19}$ and AIST $^{20}$ ). A nonArrhenius behaviour was observed in the Kissinger plots for GST in these measurements which cannot be described with a 
single activation energy. The analysis of Kissinger plots is normally carried out using the JMAK theory assuming mainly growth dominated crystallization. ${ }^{16,42}$

Here, however, we instead use the Master equation approach that includes both nucleation and growth processes and is capable of simulating complete Kissinger plots from consecutive crystallization simulations at increasing heating rates. Typical crystallization simulations using the Master rate equation are presented in Fig. 6(a) at increasing heating rates, while Fig. 6(b) illustrates the time derivatives of the crystallization curves $(\mathrm{d} \chi / \mathrm{d} t)$ to simulate DSC traces, ${ }^{42}$ which enable the identification of the peak crystallization temperatures. Figure 6 shows a progressive increase in the peak crystallization temperature $T_{p}$ and a decrease in the slope of the crystallization curves at the transition temperatures with the increasing heating rate, which is normally observed experimentally as the shift in the peaks of the DSC traces and broadening of their distribution in differential calorimetry measurements. The calculated crystallization temperatures $T_{p}$ from the Master equation simulations at different heating rates in Fig. 6 can thus be used to produce complete theoretical Kissinger plots and compare to experimental measurements.

Due to the relatively low computational cost of solving the Master rate equation system and the ability to simulate both nucleation and growth, an iterative numerical approach
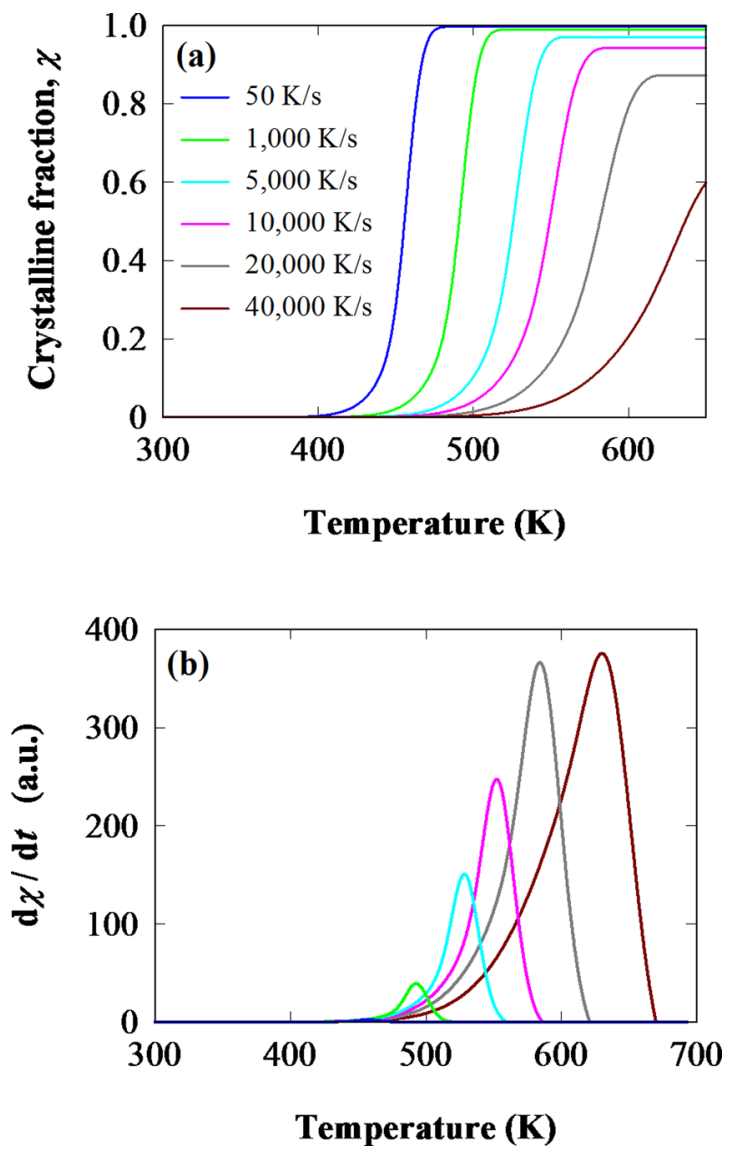

FIG. 6. (a) Calculated crystalline volume fraction as a function of temperature during ramped annealing at different heating rates. (b) Differentiated crystalline fraction curve [colour designation follows the legend of (a)]. The simulations parameters include $T_{g}=383 \mathrm{~K}$ and $m=23$. has been developed to compare simulated and experimental Kissinger plots from ultrafast DSC measurements. This is to understand the crystallization process at high heating rates and enable the extraction of the important viscosity parameters from a more detailed theoretical approach. Preliminary focus will be on implementing the iterative algorithm to extract the fragility index parameter $m$ for the MYEGA viscosity model from ultrafast DSC experimental measurements reported for GST in Ref. 16. In this case, it is assumed that the glass transition temperature is constant (within the range of published values in Table I) for all the simulated heating rates, in accordance with the procedure used in the literature to analyse Kissinger plots. ${ }^{16,19}$ The iterative algorithm proceeds by carrying out a complete crystallization simulation using ramped annealing at one heating rate from the solution of the Master equation system, using a starting value of $m=17$ for the fragility. The simulated crystallization curve is then differentiated in time, and the computed peak temperature $T_{p}$ (corresponding to the maximum crystallization rate) is compared with the experimental value at the same heating rate (see Fig. 7) ${ }^{16,19}$ and the absolute percentage error between the two temperatures is calculated. Repeated simulations at this
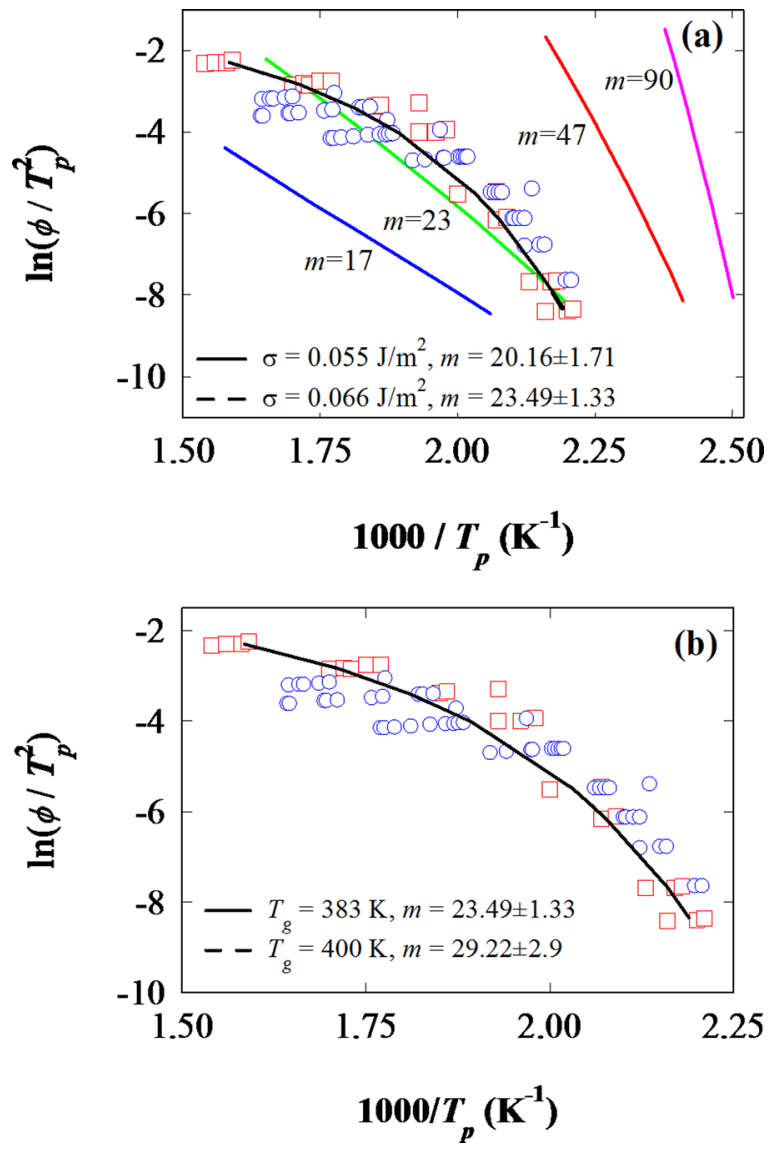

FIG. 7. Experimental Kissinger plots for GST using ultrafast DSC measurements from the study by Orava et al. (red squares) ${ }^{16}$ and simulated plots using the iterative numerical algorithm based on the Master rate equation with the fragility index being the fitting parameter. All the fitting was carried out on the experimental data of Orava et al. ${ }^{16}$ (Chen et al. ${ }^{19}$ data-blue circles-shown for consistency). (a) Fitting taking into account the uncertainty in surface energy with $T_{g}=383 \mathrm{~K}$ and showing simulated Kissinger plots using four constant fragility values for comparison and (b) fitting for two glass transition temperature values with $\sigma=0.066 \mathrm{~J} / \mathrm{m}^{2}$. Simulation parameters are listed in Table I. 
heating rate are then carried out to increment $m$ in each iteration until the absolute percentage error of the difference between the experimental and theoretical $T_{p}$ is less than $0.5 \%$. This whole process is repeated at each heating rate to produce the theoretical Kissinger plot that closely fits the experimental curve as shown in Fig. 7 (solid and dashed black lines), and the extracted values of fragility that provide best agreement at each heating rate are recorded as shown in Table II as an example. Also shown in Fig. 7 for comparison are simulated Kissinger plots using four constant values of fragility (where $m=17$ corresponds to the Arrhenius temperature dependence of viscosity).

To investigate the effects of the uncertainty in some of the modelling parameters, including the interfacial surface energy $\sigma$ and glass transition temperature $T_{g}$, on the extracted fragility values, these parameters were varied within the range of values listed in Table I in the iterative algorithm to produce the theoretical Kissinger plots shown in Fig. 7. It can be observed in Fig. 7(a) that increasing the interfacial energy $\sigma$ from 0.055 to $0.066 \mathrm{~J} / \mathrm{m}^{2}$ increases slightly the average extracted fragility values from $m \approx 20.16 \pm 1.7$ to $m$ $\approx 23.49 \pm 1.3$, respectively. It can also be observed that increasing the glass transition temperature $T_{g}$ from $383 \mathrm{~K}$ to $400 \mathrm{~K}$ (within accepted values in the literature) also increases slightly the extracted fragility values from $m \approx 23.49 \pm 1.81$ to $m \approx 29.22 \pm 2.9$, respectively, as indicated in Fig. 7(b). In general, the effect of uncertainty in $\sigma$ and $T_{g}$ on the extracted fragility values is modest, and the average value of fragility extracted from the experimental measurements using the iterative algorithm is $m \sim 23$ (assuming constant $T_{g}$ ). Moreover, no clear trend was found in the variations of $m$ with the increasing heating rate in the iterative algorithm as indicated in Table II. This extracted value of fragility is lower than the value obtained from fitting using the JMAK model of $m \sim 90$ in Ref. 16. Exploration of the outcomes of the Master equation simulations and their interpretation in the light of published experimental measurements is discussed next.

\section{DISCUSSION}

The ability of the Master equation method to model transient crystallization including nucleation and growth and its low computational cost permitted the simulation of complete Kissinger plots in this work over a wide range of heating rates and the development of an iterative algorithm for

TABLE II. Simulated Kissinger data and fitted fragility indices for $\sigma=0.066 \mathrm{~J} / \mathrm{m}^{2}$ and $T_{g}=383 \mathrm{~K}$.

\begin{tabular}{lccc}
\hline \hline $\begin{array}{l}\text { Heating } \\
\text { rate }(\mathrm{K} / \mathrm{s})\end{array}$ & $\begin{array}{c}\text { Measured } \\
T_{\mathrm{p}}(\mathrm{K})(\text { Ref. 16) }\end{array}$ & $\begin{array}{c}\text { Fitted } T_{\mathrm{p}} \\
(\mathrm{K})\end{array}$ & $\begin{array}{c}\text { Extracted } \\
\text { fragility index } m\end{array}$ \\
\hline 50 & 456.3 & 456.8 & 21.99 \\
100 & 462.3 & 463.0 & 23.18 \\
500 & 481 & 481.7 & 24.78 \\
1000 & 491.5 & 492.5 & 25.04 \\
5000 & 527.1 & 528.1 & 24.63 \\
10000 & 550.5 & 551.8 & 23.92 \\
20000 & 582.7 & 584.0 & 22.95 \\
40000 & 629.5 & 631.1 & 21.42 \\
\hline \hline
\end{tabular}

the extraction of the important viscosity parameters from published experimental measurements. The discussion will now focus on the extracted fragility index in this work using the Master rate equation in relation to previously published values and the important role of the glass transition temperature and its dependence on the heating rate on the extracted fragility values.

Extracted values of the fragility index for GST in the literature varied considerably from 20 to 140 . This variation in reported values may be attributed to several factors such as different sample and substrate structures (powder, thin-films, and flakes), sample preparation conditions, measurement technique (DSC, mechanical stress, and crystal growth velocity), pre-annealing conditions, doping, and heating and cooling rates. An equally important factor that affects the extracted values from measurements is the crystallization and viscosity models employed in the fitting and their parameters. The average fragility value of $m \sim 23$ derived in this work from fitting to the ultrafast DSC measurements is lower than reported values for GST ( $m \sim 90$ in Ref. 16). This value was computed using a more physically realistic crystallization model which incorporates both transient nucleation and growth processes. The JMAK model, which assumes stationary nucleation and growth dominated crystallization, was used to extract the fragility values for GST in Ref. 16, which is commonly classified as a nucleation dominated material. ${ }^{40}$ Moreover, in using the JMAK model for fitting to the experimental Kissinger curves in the literature, both the number of pre-existing nuclei and temperature dependant growth rate were combined into a single fitting kinetic coefficient, and the influence of each of these factors acting independently on the fitting process or on the computed fitted parameters was not clarified. Furthermore, the more physically realistic MYEGA model for the viscosity dependence on temperature was implemented here in the Master equation simulations, which has been shown to produce different results from the Cohen and Grest model employed in Ref. 16 to fit to experimental Kissinger curves and extract the fragility index.

In further exploring the potential causes for the difference between the extracted fragility values in this work and from the literature, it is fundamentally important to emphasise that the iterative algorithm used here produced complete transient crystallization simulations and extracted a separate fragility value at each heating rate. This is unlike DSC simulations employing the JMAK equation where a single fragility index was extracted over the whole range of heating rates (using a fixed value for $T_{g}$ ). This raises the important question of the validity of assuming a fixed viscosity and kinetic behaviour to describe crystallization in the phase-change material at different heating rates. In particular, it has been shown that the glass transition temperature, due to its kinetic nature, is sensitive to the heating rate and increases with the increasing heating rate in amorphous GeTe alloys, ${ }^{43}$ Chalcogenide glasses, ${ }^{44}$ and the GST phase-change material. ${ }^{17,37}$ At relatively low heating rates, the dependence of the glass transition temperature on the heating rate in GST was described by the Moynihan relation $d \ln (\phi) / d\left(1 / T_{g}\right) \approx-E / k_{B},{ }^{45}$ with a relaxation activation energy $E$ corresponding to the activation energy for shear viscous flow. ${ }^{17}$ 
To elucidate the effect of the dependence of the glass transition temperature on the heating rate in the Master equation simulations, $T_{g}$ was allowed to vary in the iterative fitting algorithm to the experimental Kissinger plots in Fig. 7 while assuming a constant value for the fragility $(m=47$ here taken for pure GST from mechanical stress measurements). ${ }^{17}$ Figure 8 (a) illustrates the extracted values of $T_{g}$ from the iterative algorithm required to achieve the closest fit to the experimental data (within $0.5 \%$ absolute difference error). This figure shows the clear trend of increasing $T_{g}$ from $416 \mathrm{~K}$ to $488 \mathrm{~K}$ when $m=47$ with the increase in the heating rate in the experiment from $50 \mathrm{~K} / \mathrm{s}$ to $40000 \mathrm{~K} / \mathrm{s}$, which is within the range of reported values for $T_{g}$ in the literature for GST as indicated in Table I. Moreover, the difference between the experimental peak crystallization temperature and fitted glass transition temperature, $T_{p}-T_{g}$ (reflecting the degree of mobility in the supercooled region), in Fig. 8(b) is approximately $40 \mathrm{~K}$ at low heating rates, in agreement with the DSC measurements for GST at low heating rates in Ref. 31. Increasing the fragility in the iterative algorithm to $m=90$ increases the diffusivity and crystallization rate, therefore reducing the peak crystallization temperature in the simulations. This requires further increases in $T_{g}$ with the increasing heating rate in the fitting algorithm to achieve the closest fit to the experimental Kissinger data in Fig. 7, as indicated in Fig. 8(a). Furthermore, the sharp drop
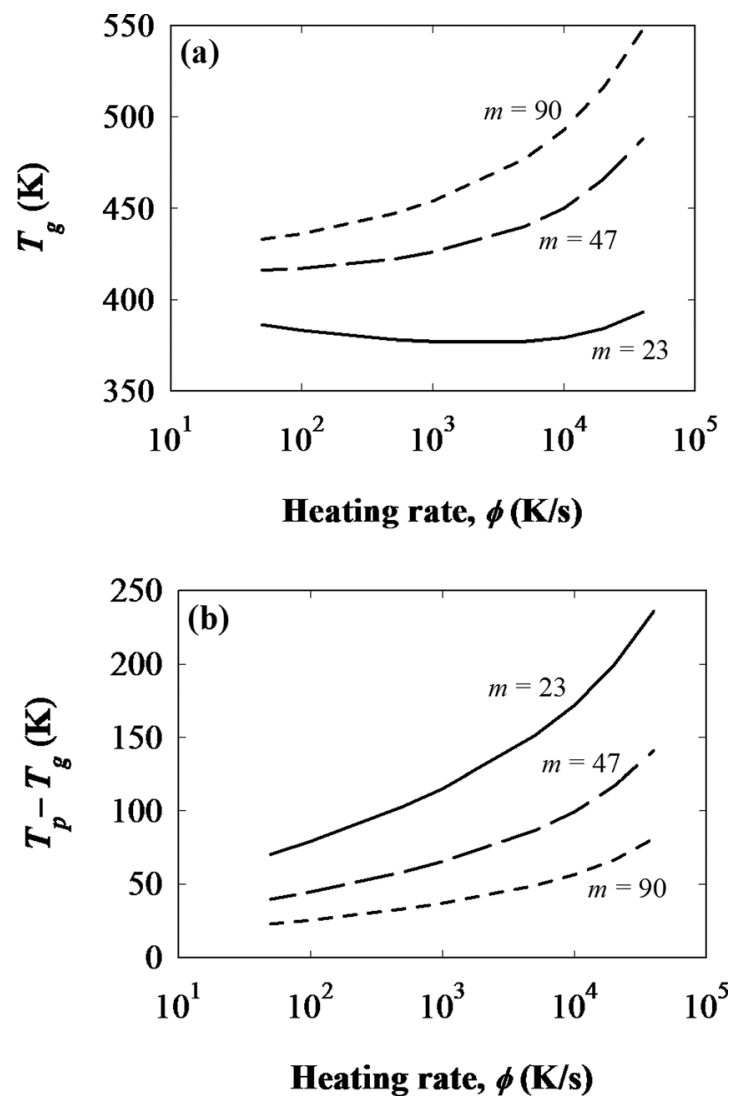

FIG. 8. (a) The glass transition temperature as a function of the heating rate determined from fitting the Master rate equation to experimental Kissinger plots, assuming constant values for the fragility. (b) The computed difference between the experimental peak crystallization temperature and derived glass transition temperature from (a). The glass transition temperatures and temperature differences for low fragility at $m=23$ are shown for comparison. of the viscosity with temperature near $T_{g}$ for $m=90$ reduces the temperature difference $T_{p}-T_{g}$ as indicated in Fig. 8(b), which at low heating rates is $\sim 22 \mathrm{~K}$ (again in agreement with Ref. 31). The increase in fitted values for $T_{g}$ for $m=90$ at high heating rates go beyond reported values for GST in the literature.

In the above simulations and fittings, the representative value of $m=47$ for the fragility index (from Ref. 17) produced values of $T_{g}$ that are in general agreement with reported values in the literature for GST. This agreement may suggest that this lower value of fragility is more reasonable than larger values found in the literature based on a constant $T_{g}$. However, this fragility value $(m=47)$ remains to be an assumption which can still be further refined to produce a modified range of values of $T_{g}$ with the increasing heating rate, particularly in the absence of experimental measurements or theory that confirms the dependence of $T_{g}$ on the heating rate for GST. Thus, the Master rate equation simulations and fittings highlight the important observations that

(a) the fragility index and glass transition parameters in the viscosity and crystallization simulations are coupled, and evaluation or extraction of one from experimental measurements requires that the other is available from the experiment or theory, and thus

(b) the accurate estimation of the fragility index from DSC measurements and Kissinger plots require information on the dependence of the glass transition temperature on the heating rate.

The simulations carried out in this work assumed the applicability of the Stokes-Einstein equation in (15) to describe the relationship between the diffusion coefficient and viscosity. Deviation from the form in the form $D \propto 1 / \eta^{\zeta}$ where $\zeta<1$ for temperatures down to $T_{g}$ was indicated using molecular dynamic simulations for the GeTe compound (not GST) in Ref. 46. This decoupling was also suggested and introduced in Ref. 16 for GST, however, mainly to overcome the inability of the Cohen and Grest viscosity model used in the analysis of Kissinger data to correctly describe the viscosity behaviour at $T_{g}$ (with the value of $10^{12} \mathrm{~Pa} \mathrm{~s}$ ). This limitation was highlighted in Refs. 25 and 20 for AIST (and indicated that the more physically realistic MYEGA models for viscosity can alternatively provide accurate analysis of DSC measurements without the need for decoupling) and Ref. 19 for GeSb. Moreover, fittings to device level measurements in Ref. 27 found no sufficient evidence for the need for this decoupling for GST. There is thus currently neither concrete evidence that confirms the breakdown of the Stokes-Einstein equation for GST nor confirmed values for the decoupling factor in the literature. Evaluation of this decoupling is also made difficult by the disparity of reported values of $T_{g}$ and fragility index for GST. Our focus here is on studying the fundamental effects of more physically realistic nucleation-growth and viscosity models and the variation in the glass transition temperature with the heating rate to explain the very good agreement that we obtained with DSC measurements at ultrafast heating rates and the subsequent alternative fitted values of fragility indices. 
The Master rate equation simulations and analysis of previously published ultrafast DSC measurements carried out in this work clarified the effects of the glass transition temperature and fragility index on the crystallization dynamics in phase-change materials. More importantly, this work highlighted the fundamental need to measure and understand the heating rate dependence of the glass transition temperature for the correct analysis of DSC measurements and estimation of the viscosity parameters necessary for modelling and characterising the crystallization dynamics in phasechange materials. This dependence will also have an impact on the estimation of crystal growth rates from DSC measurements for phase-change materials (since the growth velocity for interface controlled growth depends on viscosity). ${ }^{6}$ Alternatively and if the fragility index is known for the phase-change material, then the iterative algorithm developed in this work can potentially be used to estimate the glass transition temperature and its dependence on the heating rate from DSC measurements.

\section{CONCLUSIONS}

The crystallization dynamics in $\mathrm{Ge}_{2} \mathrm{Sb}_{2} \mathrm{Te}_{5}$ were modelled using the Master rate equation approach which includes both nucleation and growth processes, under ramped annealing with heating rates up to $4 \times 10^{4} \mathrm{~K} / \mathrm{s}$. The temperature dependence of viscosity was implemented in this numerical approach using the physically founded MYEGA model. The influence of the viscosity model parameters including the fragility index, glass transition temperature, and infinite temperature viscosity on the crystallization dynamics was investigated for different heating rates. The relatively low computational cost of solving the Master rate equation for a practical system enabled the use of a more rigorous crystallization model for the analysis of previously published ultrafast DSC measurements of the $\mathrm{Ge}_{2} \mathrm{Sb}_{2} \mathrm{Te}_{5}$ phase-change material and the development of an iterative numerical algorithm to extract the viscosity parameters from the measurements. In particular, we have taken into account the dependence of the glass transition temperature on the heating rate in the analysis of the experimental measurements and demonstrated its impact on the extracted fragility values. The outcomes of this theoretical investigation highlighted the coupling between the fragility index and glass transition temperature in the viscosity and crystallization models and the need for the experimental or theoretical determination of the dependence of glass transition temperature on the heating rate for the accurate estimation of the viscosity parameters from experimental measurements. This enables the accurate modelling and characterisation of phase-change materials and provides deeper understanding of the crystallization dynamics necessary for the development of high data rate phase-change memories and devices.

\section{ACKNOWLEDGMENTS}

The authors would like to acknowledge the financial support from the Ministry of Higher Education of Iraq for the Ph.D. scholarship of Mr. Aladool.
${ }^{1}$ Phase-Change Materials: Science and Applications, edited by S. Raoux and M. Wuttig (Springer Science \& Business Media, 2010).

${ }^{2}$ P. K. Khulbe, E. M. Wright, and M. Mansuripur, J. Appl. Phys. 88, 3926 (2000).

${ }^{3}$ W. A. Johnson and R. F. Mehl, Trans. AIME 135, 416 (1939).

${ }^{4}$ M. Avrami, J. Chem. Phys. 7, 1103 (1939).

${ }^{5}$ D. Kashchiev, Nucleation: Basic theory and applications, Part 2: Kinetics of Nucleation (Butterworth-Heinemann, Oxford, 2000), p. 113.

${ }^{6} \mathrm{~J}$. W. Christian, The Theory of Transformations in Metals and Alloys: Part I (Elsevier Science Ltd., Oxford, 2002).

${ }^{7}$ S. Raoux, W. Welnic, and D. Ielmini, Chem. Rev. 110, 240 (2010).

${ }^{8}$ M. Terao, T. Morikawa, and T. Ohta, Jpn. J. Appl. Phys. 48, 80001 (2009).

${ }^{9}$ K. F. Kelton, J. Non-Cryst. Solids 163, 283-296 (1993).

${ }^{10}$ K. B. Blyuss, P. Ashwin, A. P. Bassom, and C. D. Wright, Phys. Rev. E Stat., Nonlinear, Soft Matter Phys. 72(1), 011607 (2005).

${ }^{11}$ S. Senkader and C. D. Wright, J. Appl. Phys. 95, 504 (2004).

${ }^{12}$ C. D. Wright, K. Blyuss, and P. Ashwin, Appl. Phys. Lett. 90, 063113 (2007).

${ }^{13}$ A. Kondratiev and A. Khvan, J. Non-Cryst. Solids 432, 366 (2016).

${ }^{14}$ J. de Guzman, An. R. Soc. Esp. Fis. Quim. 11, 353 (1913).

${ }^{15}$ A. Redaelli, A. Pirovano, A. venuti, and A. L. Lacaita, J. Appl. Phys. 103, 111101 (2008).

${ }^{16}$ J. Orava, L. Greer, B. Gholipour, D. Hewak, and C. Smith, Nat. Mater. 11, 279 (2012).

${ }^{17}$ J.-Y. Cho, D. Kim, Y.-J. Park, T.-Y. Yang, and Y.-Y. Lee, Acta Mater. 94, 143 (2015).

${ }^{18}$ M. H. Cohen and G. Grest, Phys. Rev. B. 20, 1077 (1979),

${ }^{19}$ B. Chen, J. Momand, P. Vermeulen, and B. J. Kooi, Cryst. Growth Des. 16, 242 (2016).

${ }^{20}$ J. Orava, D. Hewak, and A. L. Greer, Adv. Funct. Mater. 25, 4851 (2015).

${ }^{21}$ G. W. Scherer, J. Am. Ceram. Soc. 75, 1060 (1992).

${ }^{22}$ I. Avramov and A. Milchev, J. Non-Cryst. Solids 104, 253 (1988).

${ }^{23}$ J. C. Mauro, Y. Yue, A. J. Ellison, P. K. Gupta, and D. C. Allan, Proc. Natl. Acad. Sci. U.S.A. 106, 19780 (2009).

${ }^{24}$ L. Battezatti and A. L. Greer, Acta Metall. 37, 1791 (1989).

${ }^{25}$ M. Salinga, E. Carria, A. Kaldenbach, M. Bornhofft, J. Benke, J. Mayer, and M. Wuttig, Nat. Commun. 4, 2371 (2013).

${ }^{26}$ P. Zalden, A. von Hoegen, P. Landreman, M. Wuttig, and A. M. Lindenberg, Chem. Mater. 27, 5641 (2015).

${ }^{27}$ A. Sebastian, M. Le Gallo, and D. Krebs, Nat. Commun. 5, 4314 (2014).

${ }^{28}$ C. Zhang, L. Hu, Y. Yue, and J. C. Mauro, J. Chem. Phys. 133, 014508 (2010).

${ }^{29}$ E. Morales-Sanchez, E. Prokhorov, A. Mendoza-Galvan, and J. GonzalezHernandez, J. Appl. Phys. 91, 697 (2002).

${ }^{30}$ M. Lankhorst, J. Non-Cryst. Solids 297, 210 (2002).

${ }^{31}$ J. Kalb, M. Wuttig, and F. Spaepen, J. Mater. Res. 22, 748 (2007).

${ }^{32}$ H. B. Singh and A. Holz, Solid State Commun. 45, 985 (1983).

${ }^{33}$ J. B. Zeldovich, Acta Physicochim. USSR 18, 1 (1943).

${ }^{34}$ D. Kashchiev, Surf. Sci. 18, 293 (1969).

${ }^{35}$ T. Nonaka, G. Ohbayashi, Y. Toriumi, Y. Mori, and H. Hashimoto, Thin Solid Films 370, 258 (2000).

${ }^{36}$ Y. Yue, J. Non-Cryst. Solids 354, 1112 (2008).

${ }^{37}$ J. Kalb, F. Spaepen, T. L. Pedersen, and M. Wuttig, J. Appl. Phys. 94, 4908 (2003).

${ }^{38}$ N. Yamada, E. Ohno, K. Nishiuchi, N. Akahira, and M. Takao, J. Appl. Phys. 69, 2849 (1991).

${ }^{39}$ K. Kohary and C. D. Wright, Phys. Status Solidi B 250, 944 (2013).

${ }^{40}$ H. E. Kissinger, Anal. Chem. 29, 1702 (1957).

${ }^{41}$ I. Friedrich, V. Weidenhof, W. Njoroge, P. Franz, and M. Wutting, J. Appl. Phys. 87, 4130 (2000).

${ }^{42}$ A. L. Greer, Acta Metall. 30, 171 (1982).

${ }^{43}$ I. Kaban, E. Dost, and W. Hoyer, J. Alloys Compd. 379, 166 (2004).

${ }^{44}$ J. M. Saiter, J. Ledru, A. Hamou, and A. Zumailan, Mater. Lett. 33, 91 (1997).

${ }^{45}$ F. S. Howell, R. A. Bose, P. B. Macedo, and C. T. Moynihan, J. Phys. Chem. 78, 639 (1974).

${ }^{46}$ G. C. Sosso, J. Behler, and M. Bernasconi, Phys. Status Solidi B 249, 1880 (2012). 